\title{
2 Informed consent and minors in a multicultural society
}

\author{
Mirko Daniel Garasic and Fabio Macioce
}

\section{Minors' participation in clinical research trials}

According to the World Medical Association's Declaration of Helsinki: "while the primary purpose of medical research is to generate new knowledge, this goal can never take precedence over the rights and interests of individual research subjects." Hence, voluntary and informed consent is a necessary condition. However, if trials need to involve persons unable to consent, the duty to protect them becomes pivotal. A strict interpretation of this duty could leave groups of vulnerable people without significant benefits and knowledge about their condition. Hence, we need to justify the involvement of minors and those unable to consent in clinical trials.

Due to the minors' incomplete physical and psychological development, their vulnerability is a preliminary question on every ethical discussion about paediatric clinical trials. Above all, there is a risk of harm because children cannot protect themselves, and official documents have highlighted this reality. Besides the risk of damage to health, the protection of children's rights and proper acquisition of informed consent could be legal and ethical in a multicultural society such as ours. We will employ a multicultural approach if it does not infringe upon fundamental human rights.

Given the low involvement rates presently, children's participation in clinical trials is considered insufficient.

The reasons for these deficits are to be found in a lack of interest on the part of the pharmaceutical industry, firstly because of the lower economic potential (smaller markets) and secondly because studies involving children are more complex, time-consuming, and expensive. This is enhanced by the fact that the conditions of trials change depending on the different stages of childhood development and the related risks are therefore more difficult to assess. ${ }^{2}$

DOI: $10.4324 / 9781003213215-4$ 
In research, it is essential to involve people who cannot consent, including children, so that they can assess benefits for their health, balanced with related risks. For these reasons, various institutional documents have highlighted the importance of informed consent, risk assessment and inclusion criteria in clinical trials on human subjects. We need to handle these issues carefully when dealing with minors because they cannot understand the technical information and freely give consent. ${ }^{3}$

First and foremost, we need to stress the necessity and utmost importance of paediatric clinical trials. Official documents request children involved in scientific research: "Growth and maturation processes, as well as certain specific diseases are unique to children. Specific consequences of medical interventions may be seen in children and may only appear long after exposure." Therefore, from an ethical perspective, their involvement in clinical research need not be viewed as a necessary evil.

In the past, many new products were not tested in children or adolescents although they were directed at diseases also occurring in childhood. In some cases, this resulted in children or adolescents being exposed to interventions that were either not effective or harmful. In general, this lack of information results in higher risks for children and adolescents from being exposed to interventions where little is known about their specific effects or safety in this population. Therefore, it is imperative to involve children. ${ }^{5}$

Nevertheless, minors' involvement in clinical research is not advised if trials can be carried out on adult subjects: research should be carried out first among less vulnerable subjects. ${ }^{6}$ About the order of involvement in research, it is often preferable to experiment on adults before children. Nonetheless, the Council for International Organizations of Medical Sciences (CIOMS) does not establish such a strict requirement because children may face different health issues, and their specific conditions may have been considered. However, older children with greater capacity to consent should be involved before younger children, except for special scientific reasons. $^{7}$

Trials involving minors are essential to test the effects of therapies and interventions or develop observational studies. ${ }^{8}$ To offer tailored and better healthcare for children, paediatric clinical trials are necessary to understand their particular physiological characteristics and health needs. CIOMS states that "children and adolescents must be included in health-related research unless a good scientific reason justifies their exclusion." Minors' condition requires a series of specific protections, above all because they 
cannot consent. However, their exclusion from trials needs to have a sound scientific basis concerning the risks and benefits of their involvement.

In line with this, the Nuffield Council on Bioethics affirms that children's welfare is an essential aspect to consider. However, this welfare should encompass possible scientific contributions that could benefit all children in the future. ${ }^{10}$ It does not imply a moral duty for children and parents to consent, but an aspect to determine what is good for children.

\section{What are the risks and benefits for minors?}

Clinical research on human subjects has allowed a significant increase in therapeutic and diagnostic opportunities. However, it is structurally uncertain because it is built on a scientific hypothesis that must be confirmed through investigation. Parents and society oversee children's protection, which requires risk and burden minimization and benefit calculation. ${ }^{11}$ Hence, researchers must minimize risks and burdens, balancing them with expected benefits for subjects and improvement of knowledge.

Risk assessment is a fundamental aspect of a research protocol. In paediatric clinical trials, it requires strict control:

Risk assessment includes the evaluation of the risk of the medicinal product tested or the control, the risk of withholding active treatment in some cases, the risk of the disease itself. Potential harms would include invasiveness and intrusiveness of research, the severity as well as seriousness of potential harms, the reversibility of adverse effects and reactions, and their preventability. The accumulation of research projects in the same population (over-studied population) is another potential harm. Multiple clinical trials in an individual should be discouraged. ${ }^{12}$

Significant risks in clinical trials are related to the health of the subjects and data reliability. Health-related risks depend on prior experiences with the intervention/product to be tested and their nature. If the risk is minimal, we can involve children, considering the benefits they may get compared to standard clinical treatment. These benefits can be direct or indirect. The direct benefit is health recovery by treating the patient's condition. The indirect benefit comes from general medical knowledge about the condition of the patient and others who are similarly affected or general knowledge that can benefit society. ${ }^{13}$ Having direct benefits is essential to justify therapeutic interventions. It is also important in clinical trials, "In scientific research projects, a potential direct benefit also plays a key role in the ethical evaluation of the trial." 14 
CIOMS adds: "When the social value of the studies with such research interventions and procedures is compelling, and these studies cannot be conducted in adults, a research ethics committee may permit a minor increase above minimal risk." 15

Usually, "minimal risk" means that the probability and magnitude of harm or discomfort anticipated in the research are not greater in and of themselves than those ordinarily encountered in daily life or during the performance of routine physical or psychological examinations or tests. However, this definition also lends itself to ambiguous interpretations.

In addition to risks, the burden of minors involved in research is more significant than adults. There could be anxieties, pain or interference in children's daily activities, such as separations from parents during the clinical trials, frequent invasive procedures or burdensome side effects. Parents are usually more focused on their children's life and health risks, but we must also consider the harmful effects of different burdens.

Healthy children should not be involved in clinical trials because they cannot provide proper informed consent. Prevention trials or vaccine trials are justified exceptions because, in these cases, they are preventive measures with a good risk/benefit ratio both for individuals and for society. However, they must be carried out following high standards of safety.

\section{Research integrity, ethics review and undue inducement}

The exploitation of people unable to consent is unacceptable, and a mandatory review by ethics committees is essential. ${ }^{16}$ We need to ensure research integrity by compliance with ethical principles and professional standards. ${ }^{17}$

Research ethics committees have an essential role in reviewing protocols and ensuring the "ethical acceptability" of the research. ${ }^{18}$ In paediatric trials, research ethics committees will need to involve children's healthcare specialists to assess the risks and burdens of envisaged procedures adequately. They need to scrutinize both the scientific and ethical aspects with ethical and peer reviews. The ethics committee or competent authorities should not allow the trial to proceed when ethical guidelines are not followed.

Parents may consult their child's physician about participating in a clinical trial. If the physician is the investigator, we need to make sure there is no undue influence and conflict of interests. Concerns about access to regular care should not influence the desire to participate in a clinical trial. If the researcher oversees the caring of minors involved in clinical trials, commitment to investigate cannot override the duty to care. The desire for successful research outcomes cannot compromise the proper treatment of the patient. ${ }^{19}$ 


\section{Children and family autonomy in a multicultural society}

When research involves ethnic minorities, informed consent and autonomy may even be more challenging and complicated. In its Report on Traditional Medicine Systems and their Ethical Implications, UNESCO's International Bioethics Committee addresses the challenge of cultural diversity in healthcare and medicine. It emphasizes that non-Western traditions may have a different approach to life, death, health and illness and so entail a different view regarding the patient, practitioner, patient/practitioner relationship, health services, and risk factors. ${ }^{20}$ In other words, it recognizes that Western medicine is grounded on a specific view of the human being and reflects a specific understanding of human life which may be different from that of the patient.

This difference must not be overestimated, of course. For instance, the relationship between health conceptions and ethnic identities is not completely clear. The definition of ethnic minority is problematic, ${ }^{21}$ and it may even be more arduous when we look at health-related issues. If we consider characteristics of culture, religion, language and traditions that make ethnic members different from the rest of the population, they also preserve a sense of solidarity of these groups. ${ }^{22}$ The relevance of some health traditions and conceptions that preserve ethnic identity is not easy to understand. Health conceptions, more than other issues, transcend the minorities' limits.

When a specific health tradition is relevant for the minority's identity, the balance between recognizing such a tradition and the defence of individual freedom and rights is highly problematic. While this question is of general relevance concerning subgroups, it becomes even more complicated when it relates to health challenges. ${ }^{23}$ This is because Western health laws (we may consider both U.S. and E.U.) are rooted in a few principles: patient autonomy, freedom of treatment, informed consent, giving a clear priority to the single individual and his/her rights over the group. ${ }^{24}$

Some barriers may become evident when ethnic subgroups take part in clinical trials. ${ }^{25}$ They include language barriers and limits to the comprehensibility of information concerning different linguistic uses, concepts of disease or ways of expressing sensations and attitudes regarding disease and well-being. Second, barriers may come from the individuals' different roles within the community, the group they belong to and the family. Finally, barriers may derive from a limited knowledge of research practices, procedures and ethical and legal constraints.

When we conceptualize the idea of preserving a young patient's "best interest," we tend to have an idealized version of what that patient would do or want us to do, which amounts to preserving; defending or increasing their values, priorities and autonomy. Indeed, there is a tendency to associate individual rights with individual autonomy and a corresponding 
uneasiness to associate the patient's interest with a non-individualistic version of autonomy. We need to pay attention to how it can create frictions in the healthcare system. We have discussed elsewhere the distinction between relational and individual autonomy. ${ }^{26}$ Here, we want to question some of the main legislative tools used in clinical research involving minors based on an individualistic understanding of autonomy.

According to O'Neill, ${ }^{27}$ the limits of the moral validity of individual autonomy lie in the implicit acceptance of independence as the most critical value among others to shape human beings. Relational autonomy grants an alternative conceptualization of freedom and self-government in a socially constituted agent who is equally committed to personal preferences, interpersonal relations and mutual dependencies. Accordingly, the exercise of patient autonomy largely depends on the resources available for the individual, institutional facilities and legal instruments.

Among these facilities and instruments, social rights are of primary importance. They provide the subject with goods and resources which make autonomy possible: education, healthcare assistance, welfare and participation of one's cultural and religious life. ${ }^{28}$ Moreover, autonomy requires the subject to be inserted in a relational context suitable for the exercise of freedom and characterized by positive relations of recognition. Autonomous choices, recognized by the subject as their own and correspond to their goals, depend on a series of supporting conditions that are normative, institutional and social (or more generically relational). ${ }^{29}$ Due to this complex interplay between personal capacities, institutional context and relational resources, autonomy is a concept that has variance in degrees. Conditions that support autonomy can strengthen or weaken it.

For example, in paediatric clinical trials, the subjects do not have full individual autonomy to become involved. This vulnerable group (minors and their families) needs to decide in a context of uncertainty. Hence, minors need appropriate support from adults, first from parents, but also researchers and society. As a result, specific protections are required. CIOMS states that before starting a paediatric clinical trial, researchers and ethics committees should safeguard that

1) a parent or a legally authorized representative of the child or adolescent has given permission; and 2) the agreement (assent) of the child or adolescent has been obtained in keeping with the child's or adolescent's capacity, after having been provided with adequate information about the research tailored to the child's or adolescent's level of maturity. ${ }^{30}$

Most official documents accept vulnerability as a significant concern. Vulnerability requires protection, but such protection can restrict the right to 
participate in decision-making and obtain benefits from clinical trials. There is a tension between the need to avoid harm and the right to be informed and make choices. The Nuffield Council on Bioethics challenges the correlation between vulnerability and childhood. It invites researchers to work in partnership with children and parents and not to only protect children "from" research. ${ }^{31}$ It means that minors must be encouraged to participate and make decisions. Their autonomy and integrity must be respected by giving importance to their views, listening to them and allowing their participation in decision-making. Once again, not everyone would necessarily agree on what their "autonomy" comprises and how to respect it genuinely. Crucial to this assessment are the roles of parents, which we will now investigate.

\section{The roles of parents}

Parental involvements are vital, from both legal and ethical points of view. They not only have the right to decide or duty to protect, but also assist and support their children's evolving autonomy. Parents should evaluate their "children's best interests," a complex concept decided on a case-by-case basis, considering their needs and rights. In the field of research involving minors, the notion of "avoidance of harm" may be more objective than that of "best interest." The "best interest" approach generally weighs each case's potential benefits and burdens. However, in clinical research, it is considered too generic, lending itself to ambiguous interpretations.

Since the participant's interest is not the only focus of clinical trials, the Nuffield Council on Bioethics sustains that parental consent to research "should be based on their confidence that participation in the proposed research is compatible with their child's immediate and longer-term interests." 32 Thus, the minors" "best interest," which is fundamental in clinical practice, does not become the only consideration in decision-making, overriding other ethical values.

Nevertheless, parents need decision-making support. In difficult situations, clinical trials involve burdens or risks. When parents need to deal with their children's illness which can be severe, the distress could compromise their judgement capacity. In chronic illnesses, children may have greater experience and capacity to understand the risks, burdens and benefits of a clinical trial than their parents.

Suppose that it is impossible to obtain parental permission in an emergency. In that case, the ethical review board can grant approval and then inform and involve parents as soon as possible. If the children can understand and decide in these circumstances, their decision should be respected. ${ }^{33}$

According to the U.S. Institute of Medicine, ethical review committees can waive parental permission in clinical trials of adolescents when 1) the 
research is essential to the health and well-being of adolescents and cannot reasonably or practically be carried out without the waiver; 2) the research involves treatments that State laws permit adolescents to receive without parental permission; 3) when the investigator has presented evidence that the adolescents are capable of understanding the research and their rights as research participants and 4) the research protocol includes appropriate safeguards to protect the adolescent's interests consistent with the risks of the trial. ${ }^{34}$

Parents cannot ask their children to become involved in clinical research without sound scientific evidence adequately evaluated by researchers and ethics committees. Even here, some axioms are not as simple as they might first appear. We mention only one example. The standard of bodily integrity, required by the Nuffield Council on Bioethics, is challenged by a substantial portion of the world with the practice of male circumcision. We cannot dwell on this very delicate matter here. ${ }^{35}$ Suffice it to say that this standard is not defended as vigorously or convincingly when applied in different contexts.

\section{Children and mature minors: different age, different issues}

As children mature in age, their capacity to understand takes on greater validity, and individual autonomy becomes important. To be a "minor" is a legal status, as the law fixes the age of adulthood conventionally. Nonetheless, to be a child or a young person is foremost an existential condition. The minors' continuous development becomes an ethical issue since there are significant differences between infants, children and youth. "What is more difficult and especially deserves 'ethical weighing' is research on children as children continually develop their ability to give consent as they grow older." ${ }^{\prime 36}$

Some groups have proposed an age-based classification. ICH distinguishes between newborns ( $0-27$ days), infants and toddlers ( 28 days-23 months), children (2-11 years) and adolescents (12-18 years). In the same document, ICH states that "any classification of the paediatric population into age categories is to some extent arbitrary," but still useful for study design. ${ }^{37}$ EMA makes no distinction between minors and children, using these terms synonymously. ${ }^{38}$ Nevertheless, they consider consent and its value according to age groups and the subjects' maturity. It is impossible to obtain valid assent from children between birth and three years of age. There is no specific indication for 3-6-years-old. For children of school age (from 6 years of age onwards), providing information and obtaining assent are recommended. From the age of 9, children are considered capable of understanding information. Adolescents are more independent and need 
respect and not only protection of their autonomy. "Assent from an adolescent who is a minor should be sought, and, where possible respected." 39 Researchers must, however, evaluate if adolescents have understood the information provided.

If clinical research implies minimal risks and burden for minors, the Austrian Bioethics Commission requires parental permission only for children under 14.

For minors aged 14 or older (mature minors), the Bioethics Commission does not envisage such a requirement as mature minors are allowed to act independently also in the case of other comparable medical measures. Group benefit research shall be enabled for this group of persons beyond the scope of the special laws. ${ }^{40}$

Without fixing a rigid age threshold, the Nuffield Council on Bioethics distinguishes three different situations when dealing with the broad concept of "childhood": 41

Case One: children who cannot give an opinion if they should take part in the research. They could be babies and very young children, or children who are temporarily unable to opine because they are unwell or unconscious.

Case Two: children who can form views and express wishes but cannot yet make independent decisions about research involvement.

Case Three: children and young people who potentially have the intellectual capacity and maturity to make decisions about participation in research but are considered minors in their local legal system.

Case One includes all children at the beginning of life. For children in Case Three, their assent is as good as informed consent.

We recommend that, where children and young people have sufficient maturity and understanding but are not yet treated as fully 'adult' by the law of their country, professionals should, wherever possible, seek consent from both the children or young people concerned, and from their parents. ${ }^{42}$

\section{According to CIOMS:}

As adolescents near the age of majority, their agreement to participate in research may be ethically (though not legally) equivalent to consent. In this situation, parental consent is ethically best considered as 
'co-consent' but legally, the adolescent's agreement remains assent. If child or adolescent participants reach the legal age of majority according to applicable law and become capable of independent informed consent during the research, their written informed consent to continued participation must be sought and their decision respected. ${ }^{43}$

The International Bioethics Committee of UNESCO states that the "criteria for the capacity to consent have included the ability to understand the issues involved in the decisions at stake, the ability to evaluate these rationally, a reasonable outcome of the decision and evidence of a decision being made." 44

Some jurisdictions recognize the status of "emancipated" minors who are not living with their parents and eventually have their own families. Emancipated minors can be married or parents themselves, so they can request the involvement of adults who are not their parents. "If an adolescent aged 16 to 18 is no longer a minor as defined in national law, or is an 'emancipated minor,' then written informed consent is required from these individuals as for any adult capable of giving consent. Under these conditions, informed consent is no longer required from the parents/legal representative, although an adolescent is still vulnerable and may require additional discussions and explanations." ${ }^{\prime 45}$

In long-term clinical trials, investigators should periodically check the minors' maturity and their capacity to consent. They should seek their assent or informed consent when appropriate or once the research subjects have reached the legal age. ${ }^{46}$

The example of emancipated minors highlights yet another evolution of individual/relational autonomy that requires greater attention. It is peculiar that the decisive factor in "elevating" emancipated minors to fully competent adults is when they have formed their own families. In other words, we give as much importance to the role of relationships (becoming accountable and responsible adults through parenthood) as the personal capacity to process information. On balance, we have consigned relational autonomy a more significant role than we might have realized.

\section{Conclusion}

Through documents of eminent health and bioethical organizations in the world, we have seen how the current conceptualization of autonomy is biased towards individualism. In the light of the increasingly multicultural societies we live in, these assumptions should not go unchallenged. We have seen some legal shortcomings of informed consent in clinical research, especially in minors. We call for further analysis and research on this topic that has made progress but still requires improvements. 


\section{Notes}

1 World Medical Association, Declaration of Helsinki (as Amended) (2013), art. 8, www.wma.net/policies-post/wma-declaration-of-helsinki-ethical-principlesfor-medical-research-involving-human-subjects/.

2 Austrian Bioethics Commission, Research on Persons without the Capacity to Consent-with Special Consideration of the Concept of Risk (2013), 34, www. oeaw.ac.at/resources/Record/990002754000504498.

3 European Medicines Agency (EMA), Guideline for Good Clinical Practice (2016), www.ema.europa.eu/en/ich-e6-r2-good-clinical-practice.

4 European Medicines Agency, Ethical Considerations for Clinical Trials on Medicinal Products Conducted with Paediatric Population (2008), 4, https://ec.europa. eu/health//sites/health/files/files/eudralex/vol-10/ethical_considerations_en.pdf.

5 Council for International Organizations of Medical Sciences (CIOMS), International Ethical Guidelines for Health-Related Research Involving Humans, Commentary on Guideline, (2016), 17, https://cioms.ch/publications/product/ international-ethical-guidelines-for-health-related-research-involving-humans/.

6 EMA (2008), 5.

7 CIOMS (2016).

8 Austrian Bioethics Commission (2013), 36.

9 CIOMS (2016).

10 Nuffield Council on Bioethics, Children and Clinical Research: Ethical Issues (2015), para 4.28, www.nuffieldbioethics.org/publications/children-and-clinical-research

11 International Conference on Harmonisation of Technical Requirements for Registration of Pharmaceuticals for Human Use-ICH, E 11: Clinical Investigation of Medicinal Products in the Paediatric Population (2000), www.ema.europa. $\mathrm{eu} / \mathrm{en} /$ documents/scientific-guideline/international-conference-harmonisationtechnical-requirements-registration-pharmaceuticals-human-use_en-1.pdf; World Health Organization (WHO), Standards and Operational Guidance for Ethics Review of Health-Related Research with Human Participants (2011), www.who.int/ethics/publications/9789241502948/en/; European Commission, Report from the Commission to the European Parliament and the Council: Better Medicines for Children:-From Concept to Reality (2013), https://ec.europa. $\mathrm{eu} /$ health/sites/health/files/files/paediatrics/2013_com443/paediatric_reportcom\%282013\%29443_en.pdf; CIOMS (2016).

12 EMA (2008), 17.

13 French National Consultative Ethics Committee for Health and Life Sciences, Ethical Issues Raised by Collections of Biological Material and Associated Information Data: "Biobanks," "Libraries," (2003), 3-5, www.ccne-ethique.fr/ sites/default/files/publications/avis077en.pdf.

14 Austrian Bioethics Commission (2013), 39.

15 CIOMS (2016).

16 Council of Europe, Committee on Bioethics-DH-BIO, Guide for Research Ethics Committee Members (2012), 40, www.coe.int/en/web/bioethics/guide-for-researchethics-committees-members.

17 European Group on Ethics in Science and New Technologies, Statement on the Formulation of a Code of Conduct for Research Integrity for Projects Funded by the European Commission (2015), https://ec.europa.eu/info/sites/default/files/ research_and_innovation/ege/research_integrity_ege_statement.pdf.

18 WHO $(2011), 12$. 
19 Nuffield Council on Bioethics (2015), xxxiii.

20 UNESCO (IBC), Report of the IBC on Traditional Medicine Systems and Their Ethical Implications (2013), 12, https://unesdoc.unesco.org/ark:/48223/ pf0000217457_eng.

21 Jennifer Jackson-Preece, "Beyond the (Non) Definition of Minority," European Centre for Minority Issues (ECMI) Brief (2014), 30, www.ecmi.de/publications/ issue-briefs/beyond-the-non-definition-of-minority.

22 Francesco Capotorti, Study of the Rights of the Persons Belonging to Ethnic, Religious and Linguistic Minorities (New York: UN, E/CN.4/Sub.2/384/Rev.1, 1979), https://digitallibrary.un.org/record/10387.

23 Leslie Green, "Internal Minorities and Their Rights," in Group Rights, ed. Judith Baker (Toronto, ON: University of Toronto Press, 1994), 71; Avigail Eisenberg, Reasons of Identity: A Normative Guide to the Political and Legal Assessment of Identity Claims (New York: Oxford University Press, 2009); Avigail Eisenberg and Jeff Spinner-Halev, "Introduction," in Minorities within Minorities: Equality, Rights and Diversity, ed. Avigail Eisenberg and Jeff Spinner-Halev (Cambridge: Cambridge University Press, 2005), 1-15.

24 Raanan Gillon, "Ethics Needs Principles:-Four Can Encompass the Rest:And Respect for Autonomy Should Be 'First among Equals'," Journal of Medical Ethics 29, no. 5 (2003): 307-312.

25 Simon Dein and Kamaldeep Bhui, "Issues Concerning Informed Consent for Medical Research among Non-Westernized Ethnic Minority Patients in the UK," Journal of the Royal Society of Medicine 98, no. 8 (2005): 354-356.

26 Mirko Daniel Garasic, Guantanamo and Other Cases of Enforced Medical Treatment:-A Biopolitical Analysis (New York: Springer, 2015); Fabio Macioce, "Between Autonomy and Vulnerability: Rethinking Informed Consent in a Relational Perspective," Notizie di POLITEIA 35, no. 134 (2019): 111-128.

27 Onora O'Neill, Autonomy and Trust in Bioethics (Cambridge: Cambridge University Press, 2002).

28 Robert Young, Autonomy: Beyond Negative and Positive Liberty (New York: St. Martin's Press, 1986); Joseph Raz, The Morality of Freedom (Oxford: Clarendon Press, 1986); Marina Oshana, "Personal Autonomy and Society," The Journal of Social Philosophy 29 (1998): 81-102; Amartya Sen, Development as Freedom (New York: Knopf. A., 1999).

29 Joel Anderson, "Disputing Autonomy: Second-Order Desires and the Dynamics of Ascribing Autonomy," Sats:-Nordic Journal of Philosophy 9, no. 1 (2008): $7-26$.

30 CIOMS (2016).

31 Nuffield Council on Bioethics (2015), para 4.5.

32 Ibid., para 4.33.

33 Ibid., para 6.35 .

34 Institute of Medicine of the U.S. National Academy of Sciences, Ethical Conduct of Clinical Research Involving Children (Washington, DC: The National Academies Press, 2004), 201. https://doi.org/10.17226/10958.

35 For further information on this, see Mirko Daniel Garasic, "In Defence of Male Circumcision," Monash Bioethics Review 31, no. 1 (2013): 60-69.

36 Austrian Bioethics Commission (2013), 44.

37 ICH (2000), 7.

38 EMA (2008), 7.

39 Ibid., 12. 
40 Austrian Bioethics Commission (2013), 46.

41 Nuffield Council on Bioethics (2015), para 4.5.

42 Ibid., recommendation 13.

43 CIOMS (2016).

44 UNESCO (IBC), Report of the IBC on Consent, no. 79 (2008), https://ethics. iarc.fr/Documents/IBC_consent.pdf.

$45 \operatorname{EMA}(2008), 10$.

46 Ibid.; International Conference on Harmonisation of Technical Requirements for Registration of Pharmaceuticals for Human Use (ICH), Addendum to E 11: Clinical Investigation of Medicinal Products in the Paediatric Population (2016), 5, www. ema.europa.eu/en/ich-e11r1-step-5-guideline-clinical-investigation-medicinalproducts-pediatric-population; Italian Committee for Bioethics, Clinical Trials in Adult or Minor Patients Who Are Unable to Give Informed Consent in Emergencies (2012), http://bioetica.governo.it/en/opinions/opinions-responses/clinicaltrials-in-adult-or-minor-patients-who-are-unable-to-give-informed-consent-inemergency-situations/; Italian Committee for Bioethics, Paediatric Biobanks (2014), 11, http://bioetica.governo.it/en/opinions/opinions-responses/pediatricbiobanks/. 